\title{
APPROXIMATION OF SOLUTIONS OF A GENERALIZED VARIATIONAL INEQUALITY PROBLEM BASED ON ITERATIVE METHODS
}

\author{
Sun Young Cho
}

\begin{abstract}
In this paper, a generalized variational inequality problem is considered. An iterative method is studied for approximating a solution of the generalized variational inequality problem. Strong convergence theorem are established in a real Hilbert space.
\end{abstract}

\section{Introduction and preliminaries}

Variational inequality problems have been found with an explosive growth in theoretical advances, algorithmic development and applications across all the discipline of pure and applied sciences, see [2-14] and the references therein. They combine novel theoretical and algorithmic advances with new domain of applications. Analysis of these problems requires a blend of technics from convex analysis, functional analysis and numerical analysis. As a result of interaction between different branches of mathematical and engineering sciences, we now have a variety of techniques to analysis various algorithms for solving variational inequalities and related optimization. It is well known that the variational inequality problems are equivalent to the fixed point problems. This alternative equivalent formulation is very important from the numerical analysis point of view. In particular, solutions of the variational inequality problems can be computed using the iterative projection methods. It is well known that the convergence of the projection method requires the operator $T$ to be strongly monotone and Lipschitz continuous. In this paper, we shall the equivalence to study a generalized variational inequality.

Throughout this paper, we always assume that $H$ is a real Hilbert space, whose inner product and norm are denoted by $\langle\cdot, \cdot\rangle$ and $\|\cdot\|$. Let $C$ be a nonempty closed and convex subset of $H$ and $A: C \rightarrow H$ a nonlinear mapping.

Received May 22, 2009.

2000 Mathematics Subject Classification. 47H05, 47H09, $47 \mathrm{~J} 25$.

Key words and phrases. variational inequality, solution, fixed point, nonexpansive mapping. 
Recall that $A$ is said to be monotone if

$$
\langle A x-A y, x-y\rangle \geq 0, \quad \forall x, y \in C .
$$

$T$ is said to be $\alpha$-strongly monotone if there exists a positive real number $\alpha$ such that

$$
\langle A x-A y, x-y\rangle \geq \alpha\|x-y\|^{2}, \quad \forall x, y \in C .
$$

$T$ is said to be $\alpha$-inverse-strongly monotone if there exists a positive real number $\alpha$ such that

$$
\langle A x-A y, x-y\rangle \geq \alpha\|A x-A y\|^{2}, \quad \forall x, y \in C .
$$

Recall that the following variational inequality problem is to find $u \in C$ such that

$$
\langle A u, v-u\rangle \geq 0, \quad \forall v \in C,
$$

which is known as the classical variational inequality introduced and studied by Stampacchia [10]. In this paper, we use $V I(C, A)$ to denote the solution set of the problem (1.1).

For given $z \in H$ and $u \in C$, we see that the following inequality holds

$$
\langle u-z, v-u\rangle \geq 0, \quad \forall v \in C
$$

if and only if $u=P_{C} z$. It is known that projection operator $P_{C}$ is nonexpansive.

One can see that the variational inequality problem (1.1) is equivalent to a fixed point problem. An element $u \in C$ is a solution of the variational inequality problem (1.1) if and only if $u \in C$ is a fixed point of the mapping $P_{C}(I-\lambda A)$, where $I$ is the identity mapping and $\lambda>0$ is a constant.

In this paper, we consider the following generalized variational inequality problem. Find $u \in C$ such that

$$
\langle u-A u+\lambda B u, v-u\rangle \geq 0, \quad \forall v \in C .
$$

We see that (1.2) is reduced to the classical variational inequality (1.1) if $A=I$, the identity mapping. In this paper, we use $V I(C, B, A)$ to denote the solution set of the problem (1.2).

Let $T: C \rightarrow C$ be a mapping. We denote by $F(T)$ the fixed point set of $T$. Recall that $T$ is said to be nonexpansive if

$$
\|T x-T y\| \leq\|x-y\|, \quad \forall x, y \in C .
$$

Recently, many authors studied the problem of finding a solution of the classical variational inequality problem (1.1) by iterative methods. Iiduka, Takahashi and Toyoda [6] obtained the following result.

Theorem ITT. Let $C$ be a nonempty closed convex subset of a real Hilbert space $H$ and let $A$ be an $\alpha$-inverse strongly monotone operator of $H$ into $H$ with $\operatorname{VI}(C, A) \neq \emptyset$. Let $\left\{x_{n}\right\}$ be a sequence defined as follows: $x_{1}=x \in C$ and

$$
x_{n+1}=P_{C}\left(\alpha_{n} x_{n}+\left(1-\alpha_{n}\right) P_{C}\left(x_{n}-\lambda_{n} A x_{n}\right)\right)
$$


for every $n=1,2, \ldots$, where $P_{C}$ is the metric projection from $H$ onto $C,\left\{\alpha_{n}\right\}$ is a sequence in $[-1,1]$, and $\left\{\lambda_{n}\right\}$ is a sequence in $[0,2 \alpha]$. If $\left\{\alpha_{n}\right\}$ and $\left\{\lambda_{n}\right\}$ are chosen so that $\left\{\alpha_{n}\right\} \in[a, b]$ for some $a, b$ with $-1<a<b<1$ and $\left\{\lambda_{n}\right\} \in[c, d]$ for some $c, d$ with $0<c<d<2(1+a) \alpha$, then $\left\{x_{n}\right\}$ converges weakly to some element of $V I(C, A)$.

In 2005, Iiduka and Takahashi [5] further studied the problem of of finding a solution of the classical variational inequality problem (1.1) by considering Halpern-type iterative methods. To be more precise, they proved the following theorem.

Theorem IT. Let $C$ be a closed convex subset of a real Hilbert space H. Let A be an $\alpha$-inverse-strongly monotone mapping of $C$ into $H$ such that $V I(C, A) \neq$ $\emptyset$. Suppose $x_{1}=x \in C$ and $\left\{x_{n}\right\}$ is given by

$$
x_{n+1}=\alpha_{n} x+\left(1-\alpha_{n}\right) P_{C}\left(x_{n}-\lambda_{n} A x_{n}\right)
$$

for every $n=1,2, \ldots$, where $\left\{\alpha_{n}\right\}$ is a sequence in $[0,1)$ and $\left\{\lambda_{n}\right\}$ is a sequence in $[a, b]$. If $\left\{\alpha_{n}\right\}$ and $\left\{\lambda_{n}\right\}$ are chosen so that $\left\{\lambda_{n}\right\} \in[a, b]$ for some $a, b$ with $0<a<b<2 \alpha$,

$$
\lim _{n \rightarrow \infty} \alpha_{n}=0, \sum_{n=1}^{\infty} \alpha_{n}=\infty, \sum_{n=1}^{\infty}\left|\alpha_{n+1}-\alpha_{n}\right|<\infty \text { and } \sum_{n=1}^{\infty}\left|\lambda_{n+1}-\lambda_{n}\right|<\infty,
$$

then $\left\{x_{n}\right\}$ converges strongly to $P_{V I(C, A)} x$.

In this paper, motivated by the above results, we consider the generalized variational inequality problem (1.2) by a Halpern-type iterative method. Strong convergence theorems are established in a real Hilbert space.

In order to prove our main results, we need the following lemmas.

Lemma 1.1 ([11]). Let $\left\{x_{n}\right\}$ and $\left\{y_{n}\right\}$ be bounded sequences in a Banach space $E$ and let $\left\{\beta_{n}\right\}$ be a sequence in $[0,1]$ with $0<\liminf _{n \rightarrow \infty} \beta_{n} \leq \limsup _{n \rightarrow \infty} \beta_{n}$ $<1$. Suppose $x_{n+1}=\left(1-\beta_{n}\right) y_{n}+\beta_{n} x_{n}$ for all integers $n \geq 0$ and

$$
\limsup _{n \rightarrow \infty}\left(\left\|y_{n+1}-y_{n}\right\|-\left\|x_{n+1}-x_{n}\right\|\right) \leq 0 .
$$

Then $\lim _{n \rightarrow \infty}\left\|y_{n}-x_{n}\right\|=0$.

Lemma $1.2([1])$. Let $C$ be a nonempty closed and convex subset of a real Hilbert space $H$ and $T: C \rightarrow C$ a nonexpansive mapping. Then $I-T$ is demi-closed at zero.

Lemma $1.3([15])$. Assume that $\left\{\alpha_{n}\right\}$ is a sequence of nonnegative real numbers such that

$$
\alpha_{n+1} \leq\left(1-\gamma_{n}\right) \alpha_{n}+\delta_{n},
$$

where $\left\{\gamma_{n}\right\}$ is a sequence in $(0,1)$ and $\left\{\delta_{n}\right\}$ is a sequence such that

(1) $\sum_{n=1}^{\infty} \gamma_{n}=\infty$;

(2) $\limsup _{n \rightarrow \infty} \delta_{n} / \gamma_{n} \leq 0$ or $\sum_{n=1}^{\infty}\left|\delta_{n}\right|<\infty$. 
Then $\lim _{n \rightarrow \infty} \alpha_{n}=0$.

Lemma 1.4. Let $C$ be a nonempty closed and convex subset of a real Hilbert space $H$. Let $A, B: C \rightarrow H$ be two nonlinear mappings. $u$ is a solution of the variational inequality if and only if $u$ is a fixed point of the mapping $P_{C}(A-\lambda B)$, where $P_{C}$ denotes the metric projection from $H$ onto $C$ and $\lambda$ is a positive constant.

Proof. Note that

$$
u=P_{C}(A-\lambda B) u \Longleftrightarrow\langle u-A u+\lambda B u, v-u\rangle \geq 0, \quad \forall v \in C .
$$

This completes the proof.

\section{Main results}

Theorem 2.1. Let $H$ be a real Hilbert space, $C$ a nonempty closed and convex subset of $H$. Let $A: C \rightarrow H$ be an $\alpha$-strongly monotone and L-Lipschitz continuous mapping and $B: C \rightarrow H$ a $\beta$-strongly monotone and $K$-Lipschitz continuous mapping such that $\operatorname{VI}(C, B, A) \neq \emptyset$. Let $\left\{x_{n}\right\}$ be a sequence generated by the following algorithm:

$$
x_{0} \in C, \quad x_{n+1}=\alpha_{n} u+\beta_{n} x_{n}+\gamma_{n} P_{C}\left(A x_{n}-\lambda B x_{n}\right), \quad n \geq 0,
$$

where $u$ is fixed element in $C, \lambda$ is a positive constant and $\left\{\alpha_{n}\right\},\left\{\beta_{n}\right\}$ and $\left\{\gamma_{n}\right\}$ are sequences in $(0,1)$. Assume that above control sequences satisfy the following restrictions:

(C1) $\alpha_{n}+\beta_{n}+\gamma_{n}=1, \forall n \geq 0$;

(C2) $\lim _{n \rightarrow \infty} \alpha_{n}=0, \sum_{n=1}^{\infty} \alpha_{n}=\infty$;

(C3) $0<\liminf _{n \rightarrow \infty} \beta_{n} \leq \limsup _{n \rightarrow \infty} \beta_{n}<1$;

(C4) $\sqrt{1-2 a+L^{2}}+\sqrt{1-2 \lambda \beta+\lambda^{2} K^{2}} \leq 1$.

Then the sequence $\left\{x_{n}\right\}$ converges strongly to $\bar{x} \in V I(C, B, A)$, where $\bar{x}=$ $P_{V I(C, B, A)} u$.

Proof. Put $W=P_{C}(A-\lambda B)$. Next, we show that $W$ is nonexpansive. Indeed, for any $x, y \in C$, we have

$$
\begin{aligned}
\|W x-W y\| & \leq\|(A x-A y)-\lambda(W x-W y)\| \\
& \leq\|(x-y)-(W x-W y)\|+\|(x-y)-\lambda(B x-B y)\| .
\end{aligned}
$$

Note that

$$
\begin{aligned}
\|(x-y)-(A x-A y)\|^{2} & =\|x-y\|^{2}-2\langle x-y, A x-A y\rangle+\|A x-A y\|^{2} \\
& \leq\|x-y\|^{2}-2 \alpha\|x-y\|^{2}+L^{2}\|x-y\|^{2} \\
& =\left(1-2 a+L^{2}\right)\|x-y\|^{2}
\end{aligned}
$$


and

$(2.3)$

$$
\begin{aligned}
\|(x-y)-\lambda(B x-B y)\|^{2} & =\|x-y\|^{2}-2 \lambda\langle x-y, A x-A y\rangle+\lambda^{2}\|B x-B y\|^{2} \\
& \leq\|x-y\|^{2}-2 \lambda \beta\|x-y\|^{2}+\lambda^{2} K^{2}\|x-y\|^{2} \\
& =\left(1-2 \lambda \beta+\lambda^{2} K^{2}\right)\|x-y\|^{2} .
\end{aligned}
$$

Substituting (2.2) and (2.3) into (2.1), we from the condition (C4) obtain that

$$
\|W x-W y\| \leq\|x-y\|,
$$

which shows that the mapping $W$ is nonexpansive.

Next, we show that the sequence $\left\{x_{n}\right\}$ is bounded. Letting $p \in V I(C, B, A)$, it follows from Lemma 1.4 that $p=W p$. Note that

$$
\begin{aligned}
\left\|x_{n+1}-p\right\| & \leq \alpha_{n}\|u-p\|+\beta_{n}\left\|x_{n}-p\right\|+\gamma_{n}\left\|W x_{n}-p\right\| \\
& \leq \alpha_{n}\|u-p\|+\beta_{n}\left\|x_{n}-p\right\|+\gamma_{n}\left\|x_{n}-p\right\| \\
& =\alpha_{n}\|u-p\|+\left(1-\alpha_{n}\right)\left\|x_{n}-p\right\| .
\end{aligned}
$$

By simple inductions, we have

$$
\left\|x_{n}-p\right\| \leq \max \left\{\left\|x_{0}-p\right\|,\|u-p\|\right\},
$$

which gives that the sequence $\left\{x_{n}\right\}$ is bounded. Put

$$
l_{n}=\frac{x_{n+1}-\beta_{n} x_{n}}{1-\beta_{n}}, \quad \forall n \geq 0 .
$$

That is, $x_{n+1}=\left(1-\beta_{n}\right) l_{n}+\beta_{n} x_{n}$. Now, we compute $\left\|l_{n+1}-l_{n}\right\|$. From

$$
\begin{aligned}
l_{n+1}-l_{n} & =\frac{\alpha_{n+1} u+\left(1-\alpha_{n+1}-\beta_{n+1}\right) W x_{n+1}}{1-\beta_{n+1}}-\frac{\alpha_{n} u+\left(1-\alpha_{n}-\beta_{n}\right) W x_{n}}{1-\beta_{n}} \\
& =\frac{\alpha_{n+1}}{1-\beta_{n+1}}\left(u-W x_{n+1}\right)-\frac{\alpha_{n}}{1-\beta_{n}}\left(u-W x_{n}\right)+W x_{n+1}-W x_{n},
\end{aligned}
$$

we obtain that

$$
\begin{aligned}
& \left\|l_{n+1}-l_{n}\right\| \\
\leq & \frac{\alpha_{n+1}}{1-\beta_{n+1}}\left\|u-W x_{n+1}\right\|+\frac{\alpha_{n}}{1-\beta_{n}}\left\|u-W x_{n}\right\|+\left\|W x_{n+1}-W x_{n}\right\| \\
\leq & \frac{\alpha_{n+1}}{1-\beta_{n+1}}\left\|u-W x_{n+1}\right\|+\frac{\alpha_{n}}{1-\beta_{n}}\left\|u-W x_{n}\right\|+\left\|x_{n+1}-x_{n}\right\|
\end{aligned}
$$

which implies that

$$
\left\|l_{n+1}-l_{n}\right\|-\left\|x_{n+1}-x_{n}\right\| \leq \frac{\alpha_{n+1}}{1-\beta_{n+1}}\left\|u-W x_{n+1}\right\|+\frac{\alpha_{n}}{1-\beta_{n}}\left\|u-W x_{n}\right\| .
$$

In view of the conditions $(\mathrm{C} 2)$ and $(\mathrm{C} 3)$, we obtain that

$$
\limsup _{n \rightarrow \infty}\left(\left\|l_{n+1}-l_{n}\right\|-\left\|x_{n+1}-x_{n}\right\|\right) \leq 0 .
$$

From Lemma 1.1, we see that $\lim _{n \rightarrow \infty}\left\|l_{n}-x_{n}\right\|=0$. It follows that

$$
\lim _{n \rightarrow \infty}\left\|x_{n+1}-x_{n}\right\|=\lim _{n \rightarrow \infty}\left(1-\beta_{n}\right)\left\|l_{n}-x_{n}\right\|=0 .
$$


Next, we show that

$$
\limsup _{n \rightarrow \infty}\left\langle u-\bar{x}, x_{n}-\bar{x}\right\rangle \leq 0 .
$$

To show it, we choose a subsequence $\left\{x_{n_{i}}\right\}$ of $\left\{x_{n}\right\}$ such that

$$
\limsup _{n \rightarrow \infty}\left\langle u-\bar{x}, x_{n}-\bar{x}\right\rangle=\lim _{i \rightarrow \infty}\left\langle u-\bar{x}, x_{n_{i}}-\bar{x}\right\rangle .
$$

As $\left\{x_{n_{i}}\right\}$ is bounded, we have that there is a subsequence $\left\{x_{n_{i_{j}}}\right\}$ of $\left\{x_{n_{i}}\right\}$ converges weakly to $q$. We may assume, without loss of generality, that $x_{n_{i}} \rightarrow q$. On the other hand, we have

$$
\begin{aligned}
\left\|x_{n}-W x_{n}\right\| & \leq\left\|x_{n}-x_{n+1}\right\|+\left\|x_{n+1}-W x_{n}\right\| \\
& \leq\left\|x_{n}-x_{n+1}\right\|+\alpha_{n}\left\|u-W x_{n}\right\|+\beta_{n}\left\|x_{n}-W x_{n}\right\|,
\end{aligned}
$$

from which it follows that

$$
\left(1-\beta_{n}\right)\left\|x_{n}-W x_{n}\right\| \leq\left\|x_{n}-x_{n+1}\right\|+\alpha_{n}\left\|u-W x_{n}\right\| .
$$

From (2.4) and the conditions (C2) and (C3), we obtain that

$$
\lim _{n \rightarrow \infty}\left\|x_{n}-W x_{n}\right\|=0 .
$$

From Lemma 1.2, we see that $q \in F(W)=V I(C, B, A)$. Thanks to (2.5), we arrive at

$$
\limsup _{n \rightarrow \infty}\left\langle u-\bar{x}, x_{n}-\bar{x}\right\rangle=\langle u-\bar{x}, q-\bar{x}\rangle \leq 0 .
$$

Finally, we show that $x_{n} \rightarrow \bar{x}$ as $n \rightarrow \infty$. Note that

$$
\begin{aligned}
& \left\|x_{n+1}-\bar{x}\right\|^{2} \\
= & \left\langle\alpha_{n} u+\beta_{n} x_{n}+\gamma_{n} W x_{n}-\bar{x}, x_{n+1}-\bar{x}\right\rangle \\
= & \alpha_{n}\left\langle u-\bar{x}, x_{n+1}-\bar{x}\right\rangle+\beta_{n}\left\langle x_{n}-\bar{x}, x_{n+1}-\bar{x}\right\rangle+\gamma_{n}\left\langle W x_{n}-\bar{x}, x_{n+1}-\bar{x}\right\rangle \\
\leq & \alpha_{n}\left\langle u-\bar{x}, x_{n+1}-\bar{x}\right\rangle+\beta_{n}\left\|x_{n}-\bar{x}\right\|\left\|x_{n+1}-\bar{x}\right\|+\gamma_{n}\left\|x_{n}-\bar{x}\right\|\left\|x_{n+1}-\bar{x}\right\| \\
\leq & \alpha_{n}\left\langle u-\bar{x}, x_{n+1}-\bar{x}\right\rangle+\left(1-\alpha_{n}\right)\left\|x_{n}-\bar{x}\right\|\left\|x_{n+1}-\bar{x}\right\| \\
\leq & \frac{\left(1-\alpha_{n}\right)}{2}\left\|x_{n}-\bar{x}\right\|^{2}+\frac{1}{2}\left\|x_{n+1}-\bar{x}\right\|^{2}+\alpha_{n}\left\langle u-\bar{x}, x_{n+1}-\bar{x}\right\rangle,
\end{aligned}
$$

which implies that

$$
\left\|x_{n+1}-\bar{x}\right\|^{2} \leq\left(1-\alpha_{n}\right)\left\|x_{n}-\bar{x}\right\|^{2}+\alpha_{n}\left\langle u-\bar{x}, x_{n+1}-\bar{x}\right\rangle .
$$

From the condition (C2), (2.6) and applying Lemma 1.3 to (2.7), we obtain that

This completes the proof.

$$
\lim _{n \rightarrow \infty}\left\|x_{n}-\bar{x}\right\|=0
$$

As applications of Theorem 2.1, we have the following results on the classical variational inequality (1.1). 
Corollary 2.2. Let $H$ be a real Hilbert space, $C$ a nonempty closed and convex subset of $H$. Let $B: C \rightarrow H$ be a $\beta$-strongly monotone and $K$-Lipschitz continuous mapping such that $V I(C, B) \neq \emptyset$. Let $\left\{x_{n}\right\}$ be a sequence generated by the following algorithm:

$$
x_{0} \in C, \quad x_{n+1}=\alpha_{n} u+\beta_{n} x_{n}+\gamma_{n} P_{C}\left(x_{n}-\lambda B x_{n}\right), \quad n \geq 0,
$$

where $u$ is fixed element in $C, \lambda$ is a positive constant and $\left\{\alpha_{n}\right\},\left\{\beta_{n}\right\}$ and $\left\{\gamma_{n}\right\}$ are sequences in $(0,1)$. Assume that above control sequences satisfy the following restrictions:

(C1) $\alpha_{n}+\beta_{n}+\gamma_{n}=1, \forall n \geq 0$;

(C2) $\lim _{n \rightarrow \infty} \alpha_{n}=0, \sum_{n=1}^{\infty} \alpha_{n}=\infty$;

(C3) $0<\liminf _{n \rightarrow \infty} \beta_{n} \leq \limsup _{n \rightarrow \infty} \beta_{n}<1$;

(C4) $\lambda K^{2} \leq 2 \beta$.

Then the sequence $\left\{x_{n}\right\}$ converges strongly to $\bar{x} \in V I(C, B)$, where $\bar{x}=P_{V I(C, B)} u$.

Proof. Putting $A=I$, the identity mapping, we can conclude the desired conclusion easily.

\section{References}

[1] F. E. Browder, Nonlinear operators and nonlinear equations of evolution in Banach spaces, Nonlinear functional analysis (Proc. Sympos. Pure Math., Vol. XVIII, Part 2, Chicago, Ill., 1968), pp. 1-308. Amer. Math. Soc., Providence, R. I., 1976.

[2] Y. J. Cho, S. M. Kang, and X. Qin, On systems of generalized nonlinear variational inequalities in Banach spaces, Appl. Math. Comput. 206 (2008), no. 1, 214-220.

[3] Y. J. Cho and X. Qin, Systems of generalized nonlinear variational inequalities and its projection methods, Nonlinear Anal. 69 (2008), no. 12, 4443-4451.

[4] _ Generalized systems for relaxed cocoercive variational inequalities and projection methods in Hilbert spaces, Math. Inequal. Appl. 12 (2009), no. 2, 365-375.

[5] H. Iiduka and W. Takahashi, Strong convergence theorems for nonexpansive mappings and inverse-strongly monotone mappings, Nonlinear Anal. 61 (2005), no. 3, 341-350.

[6] H. Iiduka, W. Takahashi, and M. Toyoda, Approximation of solutions of variational inequalities for monotone mappings, Panamer. Math. J. 14 (2004), no. 2, 49-61.

[7] M. A. Noor and Z. Huang, Some resolvent iterative methods for variational inclusions and nonexpansive mappings, Appl. Math. Comput. 194 (2007), no. 1, 267-275.

[8] M. A. Noor, Some developments in general variational inequalities, Appl. Math. Comput. 152 (2004), no. 1, 199-277.

[9] X. Qin and M. A. Noor, General Wiener-Hopf equation technique for nonexpansive mappings and general variational inequalities in Hilbert spaces, Appl. Math. Comput. 201 (2008), no. 1-2, 716-722.

[10] G. Stampacchia, Formes bilinéaires coercitives sur les ensembles convexes, C. R. Acad. Sci. Paris 258 (1964), 4413-4416.

[11] T. Suzuki, Strong convergence of Krasnoselskii and Mann's type sequences for oneparameter nonexpansive semigroups without Bochner integrals, J. Math. Anal. Appl. 305 (2005), no. 1, 227-239.

[12] W. Takahashi and M. Toyoda, Weak convergence theorems for nonexpansive mappings and monotone mappings, J. Optim. Theory Appl. 118 (2003), no. 2, 417-428.

[13] R. U. Verma, Generalized system for relaxed cocoercive variational inequalities and projection methods, J. Optim. Theory Appl. 121 (2004), no. 1, 203-210. 
[14] General convergence analysis for two-step projection methods and applications to variational problems, Appl. Math. Lett. 18 (2005), no. 11, 1286-1292.

[15] H. K. Xu, Iterative algorithms for nonlinear operators, J. London Math. Soc. (2) 66 (2002), no. 1, 240-256.

Department of Mathematics

Gyeongsang National University

JiNJU 660-701, KOREA

E-mail address: ooly61@yahoo.co.kr 